\title{
Comparative Analysis of Optical Coherence Tomography and Ultrasound Biomicroscopy Imaging Diagnosis of Anterior Segment of Keratoconus in Acute Stage
}

yun zhao ( $\nabla$ zhaoyunbaobei@163.com )

The Treatment Centre of Ocular Disease of Hebei Province jinxi guo

The Treatment Centre of Ocular Disease of Hebei Province jianmin wang

the Second Hospital of Hebei Medical University( jianfeng xu

The Treatment Centre of Ocular Disease of Hebei Province ming xie

The Treatment Centre of Ocular Disease of Hebei Province wei wei

The Treatment Centre of Ocular Disease of Hebei Province

\section{Research Article}

Keywords: Optical coherence tomography of anterior segment of eye, Ultrasound biomicroscopy, Keratoconus, Corneal edema

Posted Date: July 20th, 2021

DOl: https://doi.org/10.21203/rs.3.rs-633410/v1

License: (c) (i) This work is licensed under a Creative Commons Attribution 4.0 International License. Read Full License 


\section{Abstract}

Background To compare and analyze the imaging differences between anterior segment optical coherence tomography (AS-OCT) and ultrasound biomicroscopy (UBM) in acute keratoconus.

Method The features of AS-OCT and UBM images of acute keratoconus patients in Hebei Eye Hospital were analyzed retrospectively.

Result From May 2016 to December 2020, 38 patients (38 eyes) with acute keratoconus were treated in Hebei Eye Hospital. Both AS-OCT and UBM images showed rupture of the posterior elastic layer of cornea, edema and thickening of stroma layer, and cracks formed in it to communicate with anterior chamber. AS-OCT image showed that corneal epithelium was intact, and 26 cases had subcutaneous effusion. UBM images showed delamination of the posterior elastic layer interface in 26 cases. Paired $t$ test showed that there was significant difference between preoperative and postoperative best corrected visual acuity in all patients $(t=11.861, P<0.05)$. There was no significant difference in preoperative and postoperative best corrected visual acuity between group 1 and group 2 by independent sample t test $(t=$ $0.751, P>0.05, \mathrm{t}=0.219, P>0.05)$. There was significant difference in the height of rupture between group 1 and group $2(t=-7.533, P<0.001)$. Pearson correlation analysis showed that there was a significant correlation between fracture size and fracture height $(r=0.766, P<0.001)$, but no correlation between fracture size and post-elastic layer delamination $(r=-0.107, P>0.05)$. No corneal perforation was found in 38 cases (38 eyes). In the healing stage after deep lamellar keratoplasty, obvious stroma scar appeared, accompanied by corneal stroma thickening and corneal flattening.

Conclusion AS-OCT and UBM can complement each other, provide accurate corneal imaging images and parameter measurement, and predict and evaluate the development of the disease.

\section{Background}

Keratoconus is a keratopathy characterized by corneal dilation, which causes the central part of the cornea to bulge forwards and become thinner into a cone shape and produces highly irregular astigmatism. The disease mostly occurs in teenagers, and progressive onset is often noticed in both eyes successively. Acute hydrops of keratoconus (ACUTE HYDROPS) is a special condition of keratoconus, and the incidence rate is about 3\%[1]. Suddenly, the vision is often decreased in the late stage of keratoconus, and the central cornea is obviously edematous and cloudy, with a large number of blisters under the skin. The obvious edematous person is the water-drop anterior process in the central cornea (Figs. <link rid="fig1">1</link>-A and 1-B). At present, there are few studies on the anatomical features of keratoconus in the acute phase, and even fewer studies on the anatomical features using comparative analysis of AS-OCT and UBM images. In this study, AS-OCT and UBM were used to find out that the images of keratoconus in the acute phase both showed corneal posterior elastic layer rupture and discontinuous echo. Locally obvious thickening of the matrix layer; The stromal layers of the corresponding parts formed vertical fractures with different sizes to communicate with the anterior 
chamber. The thickness of the stromal layer was thin, and no definite perforation was seen. AS-OCT image can show corneal epithelium; The UBM image shows the interfacial delamination of the corneal posterior elastic layer, considering the possibility of separation of the corneal posterior elastic layer from the endodermis.

\section{Objects And Methods}

1.1 Subjects: A retrospective analysis was performed on 38 cases of keratoconus diagnosed as acute phase clinically in Hebei Eye Hospital from May 2016 to December 2020.

1.2 Criteria for case inclusion and exclusion [2]: The inclusion criteria were as follows: (1) Patients in the keratoconus group were diagnosed according to the diagnostic criteria of Rabinowitz[3]; (2) The posterior elastic layer of the cornea of the affected eye is ruptured; (3) The affected eye underwent anterior segment OCT and UBM simultaneously. At the same time, those who met the above inclusion criteria but had one of the following conditions were excluded: (1) The affected eye was accompanied by other ocular complications; (2) Accompanied by systemic diseases; (3) The affected eye has a history of eye surgery (after corneal transplantation, etc.); (4) The affected eye has a history of ocular trauma; (5) The affected eye is accompanied by clear eye infection; (6) There is no contraindication to UBM in the affected eye; (7) Only UBM or anterior segment OCT examination was performed in the affected eye. 1.3 Study Object Grouping and Methods:All the patients were divided into two groups, in which group 1 was the corneal posterior elastic layer rupture diameter $<2 \mathrm{~mm}$, and group 2 was the rupture port $>2 \mathrm{~mm}$. Routine eye examinations as well as AS-OCT and UBM were performed in both groups.

Detection methods: (1) AS-OCT(Visant OCT: Carl Zeiss) scanning parameters: $1310 \mathrm{~nm}$ infrared ray was used to scan the anterior segment of the eye at 360 degrees, with the axial scanning speed of 2000 points per second, the lateral scanning resolution of 60 microns, the axial resolution of 18-25 microns, the scanning frequency of $4 \mathrm{kHz}$, the image acquisition time of $0.125 \mathrm{~s}$ and the scanning depth of $6 \mathrm{~mm}$. The patient sat upright in front of the AS-OCT instrument in natural light, with his lower jaw placed on the jaw rest. The anterior yellow gaze marker was fixed, and the appropriate corneal scanning mode was selected. The AS-OCT instrument automatically completed the scanning, and the scanned images with better image definition and location were selected to complete the corresponding data measurement and stored. (2)UBM (Tianjin Meda) nominal ultrasonic working frequency: $50 \mathrm{MHz} ; \mathrm{Gain}$ : $\geqq 105 \mathrm{~dB}$; Resolution: axial not more than $0.04 \mathrm{~mm}$, lateral not more than $0.04 \mathrm{~mm}$. Digital scan converter (DSC) capacity: 1024×768×8bit×64; Gray scale: 256 levels; Focal length: 9 mm; Window display (width $\times$ depth):

$8.25 \mathrm{~mm} \times 5.5 \mathrm{~mm}$ (narrow view mode) Delay depth $3 \mathrm{~mm}, 16.5 \mathrm{~mm} \times 11 \mathrm{~mm}$ (panoramic mode) Delay depth $6 \mathrm{~mm}$. The patient lay flat on the hard board bed in the indoor natural light, and the appropriate optic cup was selected and placed in the eyelid of the patient. An appropriate amount of sterile normal saline was poured into the eyelid, and the UBM probe was placed in a suitable position in the eye cup for image scanning. The scanned images with good image definition and position were collected to complete the corresponding data measurement and stored. 
1.4 Statistical analysis:Statistical analysis was performed using SPSS23.0 statistical software. The enumeration data were expressed as percentages, and the measurement data of normal distribution were expressed as mean addition and subtraction of standard deviation $(\mathbb{Z X \pm S})$. The one-way analysis of variance(one-way ANOVA) was used for intra-group comparison, and paired t-test was used for intergroup comparison. Test level: $\mathrm{a}=0.05$.

\section{Results}

2.1 General results There were 33 males and 5 females. 18 in the right eye and 20 in the left eye. The maximum age was 34 years old and the minimum age was 11 years old, with an average age of 21.2 years old. Vision: there were 12 cases with manual operation at present, 19 cases with immediate index, and 7 cases with $0.01-0.1$, none of which was improved after refraction correction. All the 38 patients presented with sudden visual acuity decline, and six of them presented with sudden visual acuity decline after hard eye rolling. Five patients presented with an acute attack of keratoconus in one eye of both eyes, one of whom had undergone corneal transplantation in the contralateral eye; Six cases had myopic history of both eyes. The AS-OCT and UBM images of keratoconus in the acute phase are characterized by corneal posterior elastic layer rupture and discontinuous echo. Locally obvious thickening of the matrix layer; The stromal layers of the corresponding parts formed vertical fractures with different sizes to communicate with the anterior chamber. The thickness of the stromal layer was thin, and no definite perforation was seen. AS-OCT images showed intact corneal epithelium and subepithelial fluid in 26 cases (Figures 1-2D, E and F); UBM images showed corneal posterior elastic layer interface stratification in 26 cases (Figures 1-4G, H and I). All patients were treated with deep lamellar keratoplasty by the same operator under general anesthesia. In the healing stage after keratoplasty, there appeared obvious stromal scar accompanied by corneal stromal thickening and corneal flattening (Figures 1-4J and K). A total of 24 cases with corneal posterior elastic layer rupture less than $2 \mathrm{~mm}$ were included, among which 13 cases had corrected visual acuity less than $0.1,9$ cases had visual acuity of $0.1-0.2$, and 2 cases had visual acuity of $0.3-0.5$ one week after surgery. There were 14 cases with the rupture of the posterior elastic layer larger than $2 \mathrm{~mm}$, including 6 cases with corrected visual acuity less than $0.1,7$ cases with 0.1-0.2, and 1 case with $0.3-0.5$ one week after surgery. 2.2 Statistical results According to paired t-test, the difference between the preoperative best corrected visual acuity and the postoperative best corrected visual acuity was statistically significant $(t=11.861, P<0.05)$ (Table 1$)$. The differences in preoperative best corrected visual acuity and postoperative best corrected visual acuity between Group 1 and Group 2 after independent sample $t$-test were not statistically significant $(t=0.751, P>0.05, T=0.219, P>0.05)$ (Table 1). The differences in the heights of the rupture ports between Group 1 and Group 2 were statistically significant $(t=-7.533, P<0.001)$ (Table 1$)$. Pearson correlation analysis showed that the size of fracture opening was significantly correlated with the height of fracture opening $(r=0.766, P<$ 0.001) (Table 1), but there was no correlation between the size of fracture opening and posterior elastic layer stratification $(r=-0.107, P>0.05)($ Table 1$)$.

\section{Discussion}


With the advancement of science and technology, there are a variety of ophthalmic examination equipment, among which UBM and AS-OCT are two important examination items. UBM scans the tissue at the depth of $5 \mathrm{~mm}$ in the anterior segment of the eye through high-frequency ultrasound (50-100 $\mathrm{MHz}$ ). In the panoramic mode, the exploration depth can reach about 6-7 mm, obtaining high-resolution and high-definition images of any section, as well as quantitative analysis of many structures in the anterior segment. As a new ophthalmic imaging device, AS-OCT uses 1310nm infrared light for scanning, which can penetrate the cornea and scleral tissue, and complete the imaging and measurement of the anterior segment anatomy and pathology after cataract, refractive surgery, glaucoma, corneal transplantation and ocular trauma in a non-contact manner, providing the basis for anterior segment analysis. The scanning angle is measured from 0 to 360 degrees at a full angle, with the maximum depth of $6 \mathrm{~mm}$. AS-OCT is a form of cross-sectional imaging based on light that does not require eye contact. Compared with UBM, AS-OCT is simpler and faster in operation, and more acceptable to patients [4]. The imaging principles of the two methods are similar. AS-OCT has higher resolution, while UBM detects biological tissues through ultrasonic waves, with stronger penetration, which is much higher than that of light waves. For a normal transparent cornea, the imaging findings of the two are not much different, but for the examination of pathological changes that affect light penetration, such as obvious edema and thickening of corneal epithelium and scar in stroma layer, UBM has advantages over AS-OCT. Many articles have reported that under the condition of normal clear cornea, there is no significant difference between the measured values of UBM and AS-OCT in the comparative study of the measurement of biological parameters in the anterior segment of the eye [5-8]. However, there are few reports on the study of UBM and OCT in the anterior segment of keratoconus in the acute phase, and the comparative analysis of the two examinations is even less.

Esteban Fuentes et al. applied anterior segment OCT to analyze the anatomical features such as the thickness of epithelial and stroma layers, the hyperreflexia of anterior elastic layer, Vogt striation, and the opacity of stroma layer of keratoconus in the acute phase, and they found that the thickening of corneal epithelial layer, thinning of stroma layer, the hyperreflexia of anterior elastic layer, and the lack of stroma scar layer are the risk factors for inducing keratoconus edema in the acute phase [2]. Wang et al. found in their study that the imaging resolution of the central and peripheral cornea by UBM was low [9], and the epithelial boundary was unclear in the case of corneal edema. In this study, the imaging features of ASOCT and UBM in acute keratoconus were corneal posterior elastic layer rupture and discontinuous echo. Localized obvious edema and thickening of the matrix layer; Vertical fissures with different sizes were formed in the matrix layer of the corresponding parts to communicate with the anterior chamber. The thickness of the matrix layer was thin, and no definite perforation was seen. AS-OCT images clearly showed corneal epithelial integrity, subepithelial effusions, and epithelial separation from the anterior elastic layer. The UBM image shows that the interface of corneal posterior elastic layer is split into two layers. As there is no hemidesmosomes between the corneal posterior elastic layer and endothelial cells, the attachment relationship between the two depends on the endothelial cells themselves permanently producing new posterior elastic layer fibers, and the endothelial cells are easily separated from the posterior elastic layer. When the posterior elastic layer is ruptured, there are usually wavy bending and 
reflecting double lines with a darker place in the middle [10]. Therefore, when the aqueous humor flows back due to the fissure formation of the matrix layer, it may break through the interface of the posterior elastic layer and separate from the endothelium (no related research report has been found in the literature yet)! ) $\otimes$ Acute edema of keratoconus often occurs just below the central part of the cornea or under the nose, so we may not find any subepithelial effusion in UBM examination of the same case, which is separated from the anterior elastic layer, but it may also be caused by different angles of incidence of ultrasonic waves. Corneal edema presented as uniform and weak reflection on AS-OCT images [11-14], and the corneal boundary was relatively clear. The near-infrared light of AS-OCT can transmit the edematous corneal stroma to the endothelial surface, anterior chamber, iris and lens for cross-sectional imaging, making up for the shortage that the posterior cornea and anterior chamber cannot be observed by slit-lamp microscope when the cornea is cloudy. As the corneal stroma layer was obviously thickened during acute corneal edema, the thickest edema area in this study was about 2.63 $\mathrm{mm}$. Considering the imaging principle of AS-OCT, the permeability of light wave was relatively weak, which may cause unclear display at the posterior part of the cornea, and no interfacial layer of corneal posterior elastic layer was found. On the other hand, AS-OCT had a higher resolution, and we observed that the posterior elastic layer rupture opening in our patient was at least about $0.1 \mathrm{~mm}$, and the basal layer rupture resulted in an increased contact area with anterior aqueous humor, which also explains the fact that acute corneal edema is less likely to occur when the posterior elastic layer is simply avulsionruptured.

Although the difference between the pre-operative best corrected visual acuity and the post-operative best corrected visual acuity in all patients with acute keratoconus studied in this group is statistically significant, the post-operative visual acuity is often poor, which is related to various factors such as the scope of corneal edema, the size and location of posterior elastic layer hiatus, and the degree of stromal opacity of the patient's cornea. All the patients were treated with deep lamellar keratoplasty under general anesthesia by the same operator. In this study, it was found that there was no statistical difference between the preoperative and postoperative best corrected visual acuity of all the groups 1 and 2 . Deep lamellar keratoplasty can reduce postoperative rejection to a great extent, reduce postoperative astigmatism and relieve the pressure of insufficient fresh corneal material. However, based on the above research, in order to achieve a better visual quality for patients, we are reminded that for patients with acute keratoconus, can partial penetrating keratoplasty be used? The size of fracture opening is significantly correlated with the height of fracture opening, while it has no correlation with the stratification of back elastic layer. AS-OCT and UBM can provide us with accurate corneal imaging and measurement of ocular biological parameters, accurate positioning and indication of the size of the posterior elastic layer hiatus, and provide a very valuable reference for clinical surgery.

Esteban Fuentes et al. found that the lack of scar in the keratoconus stromal layer might be an inducing factor for the acute edema of keratoconus [2]. Due to the small number of cases in this study and the failure to observe the pre-acute corneal edema image of our patient, as well as the lack of research on its influencing factors, we will strengthen the observation of relevant factors in future studies. The stroma fissure in keratoconus at the acute stage is rarely seen under slit-lamp microscope, and it has been only 
rarely reported that in our case AS-OCT and UBM examinations both showed stroma fissure of different sizes communicating with the anterior chamber. There are two causes for it, primary and secondary. The primary one is caused by the cracking of corneal stroma layer itself, and structural change and fissure appearance are caused by corneal deformation. Secondary because of rupture of the back elastic layer. The corneal edema is aggravated by the fissures in the stroma layer, because the contact area between the stroma layer and anterior aqueous humor is enlarged due to the fissures. The fissures in the stroma layer also affect the healing of the posterior elastic layer and the recovery of corneal edema [15-17]. In our case, the matrix fractures were more clearly demonstrated on AS-OCT images than on UBM images, with clearer margins. When corneal perforation occurs due to the thin corneal stroma of patients, UBM, as a contact test, is not suitable. Compared with UBM, AS-OCT is simple and fast to operate, and easy to be accepted by patients.

\section{Conclusion}

AS-OCT and UBM can complement each other to provide accurate corneal imaging and parameter measurement for predicting and evaluating the development of the disease.

\section{Abbreviations}

UBM: ultrasound biomicroscopy; AS-OCT: anterior segment optical coherence tomography

\section{Declarations}

\section{Acknowledgments}

Not applicable

\section{Authors' contributions}

Zhao Yun and Wang Jianmin designed and analyzed the study. Guo Jinxi, Xu Jianfeng and Xe Ming carried out the measurements. Zhao Yun wrote the manuscript with support from Wang Jianmin and Guo Jinxi, Xe Ming and Wei Wei helped interpretation of data. All authors have read and approved the manuscript.

\section{Funding}

Not applicable.

\section{Availability of data and materials}

The datasets generated and/or analysed during the current study are not publicly available due to the prevision of further publications coming soon,but are available from the corresponding author on reasonable request.

\section{Ethics approval and consent to participate}


All performed procedures in this study were done according to the ethical standard of The Heibei Eye Hospital of Chinese and adhered to the 1964 Helsinki declaration and its latest amendments.

\section{Consent for publication}

Not applicable.

\section{Competing interests}

The authors declare that they have no competing interests

\section{References}

1. Zheng Zk, Liu H, Hu ZL. Anterior segment OCT examination ofacute hydrops in patients with keratoconus[J]. International Journal of Ophthalmology, 2012,12 (3): 548-549.

2. Esteban F.Otman S.Mohamed E.et al.Anatomic predictive factors of acute corneal hydrops in keratoconus an optical coherence tomography study. Ophthalmology.2015,122(8):1653-1659.

3. Rabinowitz YS.Keratoconus[J].SurvOphthalmol,1998,42(4):297-319.

4. Zhao Y, Guo J, Wei W. Comparison of intraocular lens position observed by ultrasound biomicroscopy and anterior segment coherent light tomography: a case report [J]. Chinese Journal of Practical Ophthalmology, 2017,35(12):1201-1202.

5. Doors M, Cruysberg LP, BerendschotTT,et a1.Comparison of central corneal thickness and anterior chamber depth measurements using three imaging technologies in normal eyes and after phakic intraocular lens implantation [J].G-raefes Arch Clin Exp Ophthalmic,2009,247(8):39-46.

6. Dada T,Sihota R,Gadiaï \& Aggarwal MandaIS,Gupta V Comparison of anterior segment optical coherence tomography and ultrasound biomicroscopy for assessment of the anterior segment [J]. $J$ Cataract Refract Surg.2007,33(5):837-40.

7. Garcia JP, Rosen R. Anterior segment imaging:optical coherence tomography VerSUS ultrasound biomicroscopy [J].Ophthalmic Surg Lasers Imaging.2008,39(6):476-84.

8. Kim HY, Budenz DL,Lee PS,Feuer WJ,Barton I.Comparison of central corneal thickness using anterior segment optical coherence tomography VS ultrasound pachymetry[J]. Am J Ophthalmic.2008, 145(2):228-32.

9. Mario AD, Pascuale, Pawan P, et al. Corneal deturgescence after Descemet stripping automated endothelial keratoplasty evaluated by Visante anterior segment optical coherence tomography [J]. Am J Ophthalmol.2009,148(1):32-7.

10. Li FM. Ophthalmology encyclopedia [M].Beijing: people' s health publishing house, 1997,722, 1330.

11. Hutchings N,Simpson TL,Hyun C,et a1.Swelling ofthe Human Cornea Revealed by High Speed,Ultrahigh Resolution Optical Coherence Tomography[J]. Invest OphthalmolVis Sci. 2010,

12. Kymionis GD,Ide T,Donaldson K Yoo SH.Diagnosis ofDonor Graft Partial Dislocation Behind the Iris ARer DSAEK with Anterior Segment OCT[J]. Ophthalmic Surg Lasers Imaging2010, 9:1-2. 
13. Tomoya N,Naoyuki M,Noriko O, et al. Ultrasound Biomicroscopic Examination of Acute Hydrops in Patients With Keratoconus[J]. American journal of ophthalmology. 2005,141(6):1134-1136.

14. Fan Lu,Suzhong Xu,Jia Qu,et al.Central comealthickness and corneal hysteresis during corneal swelling induced by contact lens wear with eye closure[J]. Am J Ophthalm01. 2007,1 43(4):6 16-22.

15. Tuft SJ,Gregory WM,Buckley RJ.Acute corneal hydrops in keratoconus[J]. Ophthalmology 1994,101(4):1738-1744.

16. Ingraham HJ,Donnenfeld ED,Perry HD.Keratoconus with spontaneous perforation of the cornea[J]. Arch Ophthalmol 1991,109(2):1651-1652.

17. Rubsamen PE, McLeish WM. Keratocons with acute hydrops and perforation:brief case report[J]. Cornea 1991,10(11):83-84.

\section{Tables}

Table.1 Statistical results

\begin{tabular}{lllllll} 
Items & Group1 & Group2 & \multicolumn{2}{c}{ intra-group } & \multicolumn{2}{c}{ inter-group } \\
\cline { 4 - 6 } & & & $\mathrm{F}$ & $\mathrm{P}$ & $\mathrm{t}$ & $\mathrm{P}$ \\
\hline Pre-operative vision & $2.20 \pm 0.56$ & $2.05 \pm 0.72$ & 0.751 & 0.458 & 11.861 & $\leq 0.001$ \\
\cline { 1 - 4 } Post-operative vision & $0.97 \pm 0.43$ & $0.95 \pm 0.24$ & 0.219 & 0.828 & & \\
\hline Rupture height & $2.01 \pm 0.44$ & $1.80 \pm 0.59$ & 1.19 & 0.242 & $/$ & $/$
\end{tabular}

\section{Figures}


1、4

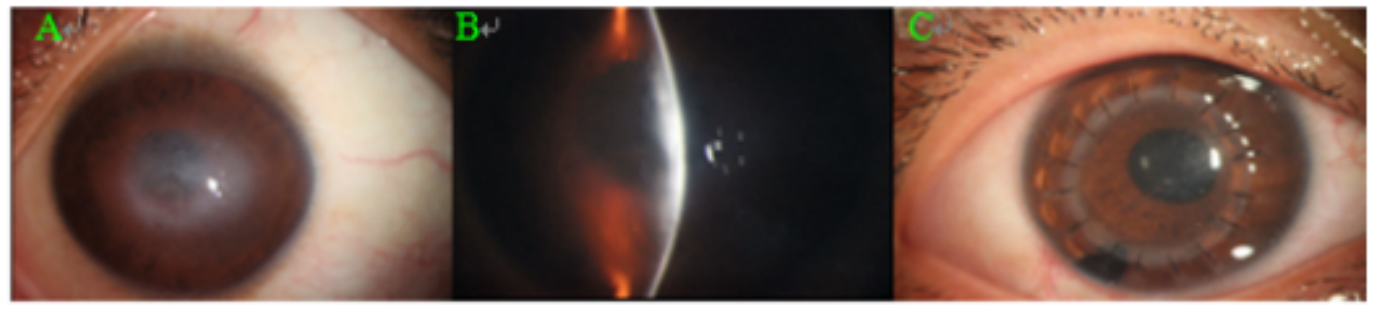

2、

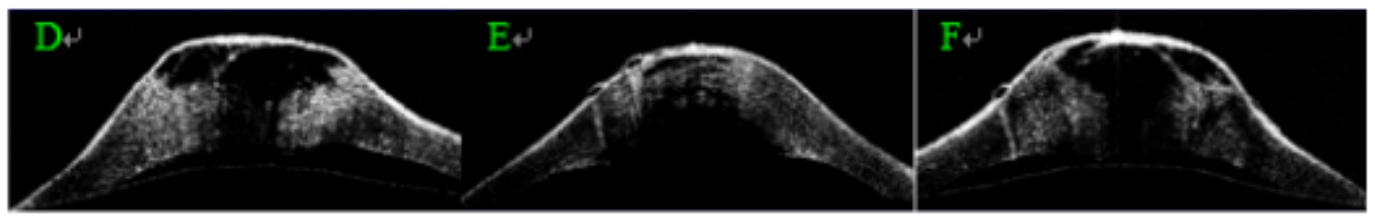

3、4

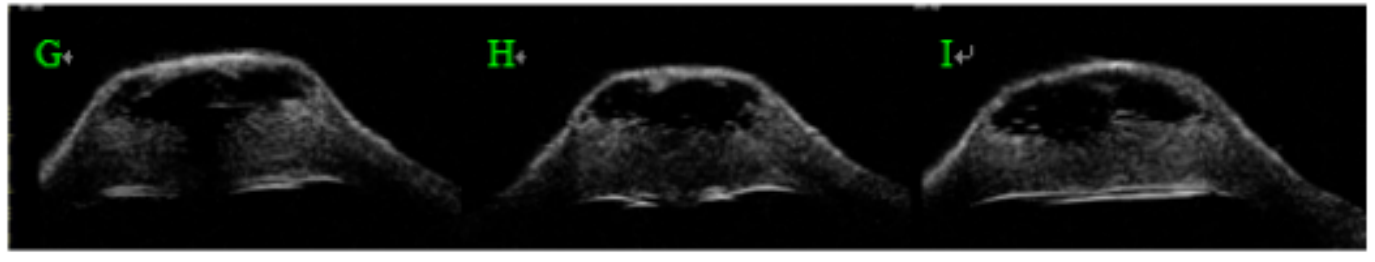

4、

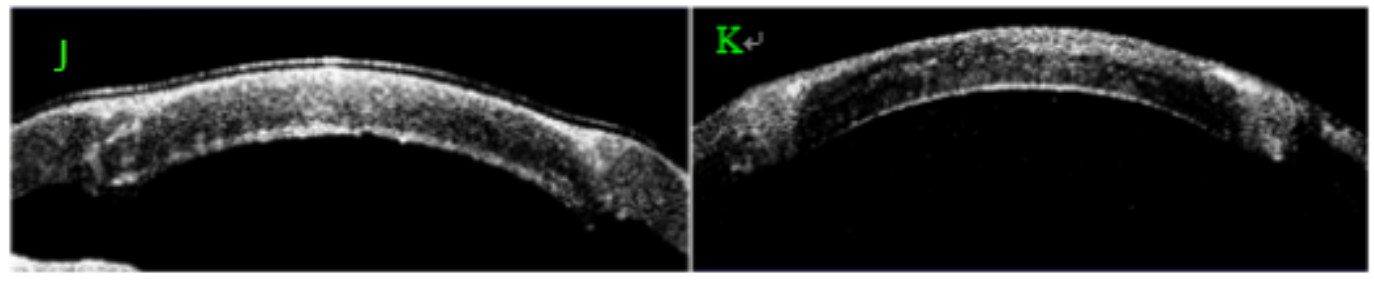

Figure 1

$1-A B C$ is photograph of the anterior segment of the eye before and after acute keratoconus transplantation; 2-DEF is an AS-OCT image of keratoconus in the acute phase. 3-GHI is an UBM image of acute keratoconus; 4-JK is an AS-OCT image after deep lamellar keratoplasty. 


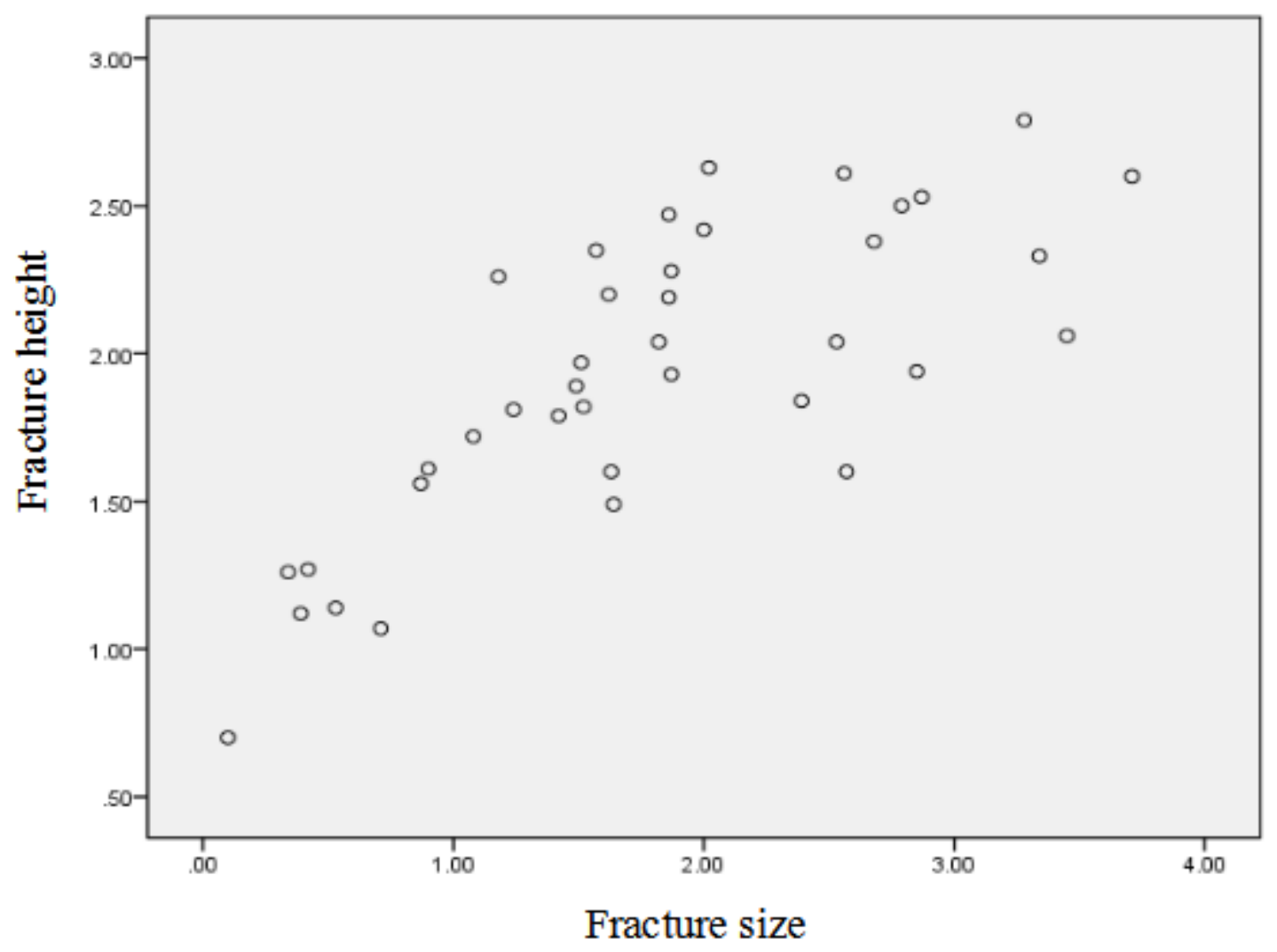

Figure 2

Correlation analysis between fracture size and fracture height 